\title{
Open anterior hip dislocation in a young adult with exposed femoral head and no neurovascular damage
}

\author{
N Muzaffar, MS Orth, A Hafeez, MBBS, N Bashir, MS Orth, S Singh, MS Orth \\ Department of Orthopaedics, Hospital for Bone and Joint Surgery, Barzalla, Srinagar, Kashmir, India
}

\begin{abstract}
Anterior hip dislocation is common in younger adults and is the result of severe, high energy trauma such as high-velocity motor vehicle accidents or falls from great heights. A similar mechanism of injury would cause a femur neck fracture in older patients. Of these, open anterior hip dislocations are extremely rare and are usually associated with other soft tissue and bony injuries. We report a case of an open anterior hip dislocation with normal neurovascular status in a third world setting.
\end{abstract}

Key Words:

trauma, open anterior, dislocation, hip, femoral head, osteonecrosis

\section{CASE REPORTS}

A young 35-year-old male labourer was involved in a high speed road traffic accident and sustained injury to his right hip and left lower leg. He was brought to the emergency department of this hospital and was preliminarily assessed as having an open anterior dislocation of the hip with the femoral head clearly visible in the inguinal region (Figure 1a and $1 \mathrm{~b}$ ). The affected limb was in gross external rotation but with no flexion or adduction and there was a contralateral open fracture of the tibia (Gustilo Anderson type III-A). The patient was in shock but the neurovascular status was intact. Hip radiographs showed an anterior-superior dislocation with no other associated bony injury (Figure 2a). The patient was resuscitated and immediately transferred to the operating room where the femoral head was relocated under anaesthesia; the reduction was checked by radiography (Figure 2b), the inguinal wound was debrided and the tibial fracture was stabilized with an external fixator. The wound was $5 \times 6 \mathrm{~cm}$ in dimension, the femoral head visible just above the inguinal ligament and the surrounding tissues were contused but the femoral vessels and nerve were intact. The wound was thoroughly irrigated with saline and debrided; primarily closed and the patient was prescribed i.v cefazolin, amikacin and metronidazole for 7 days and skin traction for 6 weeks. The wound healed satisfactorily and the tibial fracture was also subsequently managed with fixator removal and a Plaster of Paris long leg cast. There was no evidence of any osteonecrosis or infection of the hip at one year follow-up (Figure 2c). The patient was subsequently lost to follow up.

\section{DISCUSSION}

The hip joint is inherently one of the most stable joints in the body and considerable force is needed to dislocate it. In today's hectic life, traumatic hip dislocations are not uncommon, especially in developing countries with ever rising vehicular traffic and poor infrastructure. Open hip dislocation remains a rare occurrence due to the bulky muscle envelope surrounding the deeply situated hip. In a large series of hip dislocations, no open dislocations were mentioned ${ }^{1}$. The majority of hip dislocations are posterior ${ }^{1}$ the incidence of anterior dislocations comprises $2 \%$ to $11 \%$ of all dislocations. Anterior hip dislocations can be associated with femoral neurovascular injury, femoral head fractures, and acetabular fractures ${ }^{1,2}$. These injuries are associated with a poorer prognosis as well as with a delay in reduction of the joint. Open anterior hip dislocation has been reported five times in children ${ }^{2-6}$ and only twice in adults ${ }^{7,8}$. In 1982, Grundy and Kumar reported a case of an open anterior-superior hip dislocation ${ }^{7}$ in which a 26 -year-old man sustained an injury with hyperextension and external rotation force to the hip when he was forced through a fence by a vehicle. The femoral head came to rest anterior and superior to the acetabulum over the pectineal eminence. The open wound was $10 \mathrm{~cm}$ in length in line with the inguinal ligament. At the one-year follow-up, there was no evidence of infection or avascular necrosis. In 2003, Lamberti and Rabin ${ }^{8}$ reported an open anterior-inferior dislocation due to a road traffic accident resulting in a $9 \mathrm{~cm}$ long wound $10 \mathrm{~cm}$ distal to the pubis communicating with the hip joint. The wound was due to progressive medial soft tissue injury secondary to the extreme rotation and displacement of the hip. This progression started with inferior capsular disruption and joint dislocation and continued to muscle and upper-medial thigh skin rupture. There was no evidence of infection or osteonecrosis at the 12-month follow up.

A review of the literature implies that the prognosis of open hip dislocation worsens with increasing severity of the 


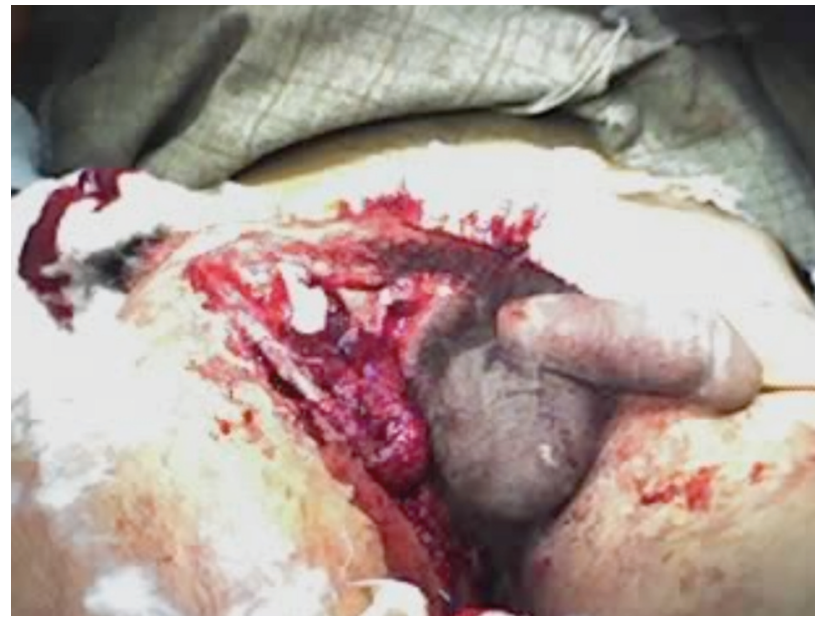

Fig. 1a: Appearance of the groin wound on presentation.

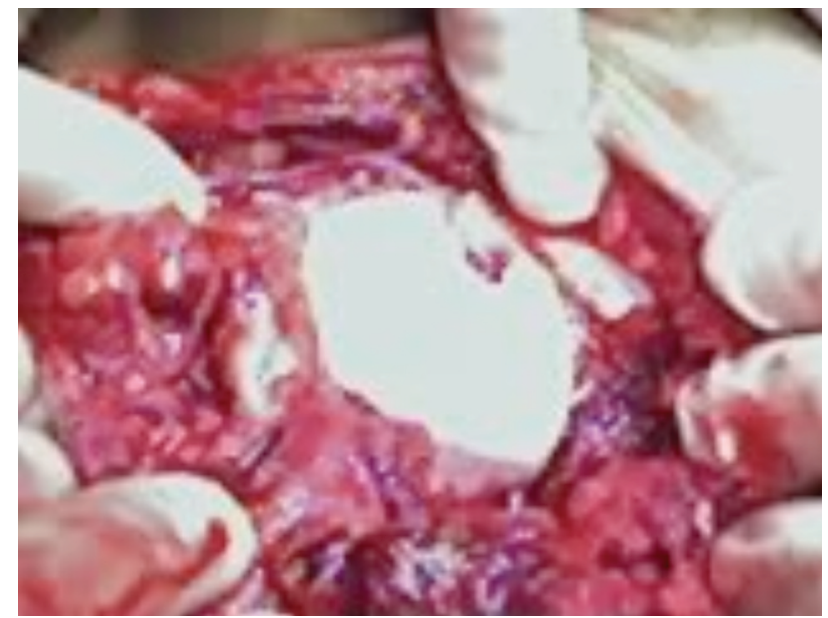

Fig. 1b: The femoral head visible anteriorly.

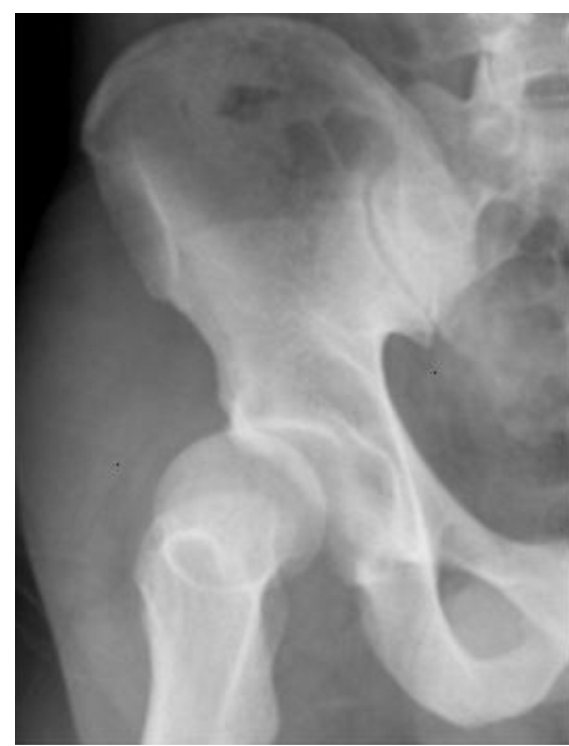

Fig. 2a: Initial radiograph showing anterior hip dislocation at admission.

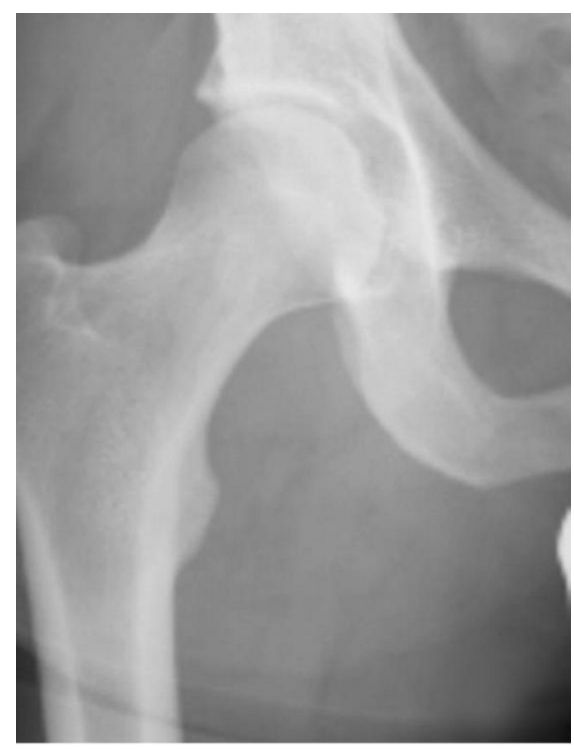

Fig. 2b: Post reduction radiograph.

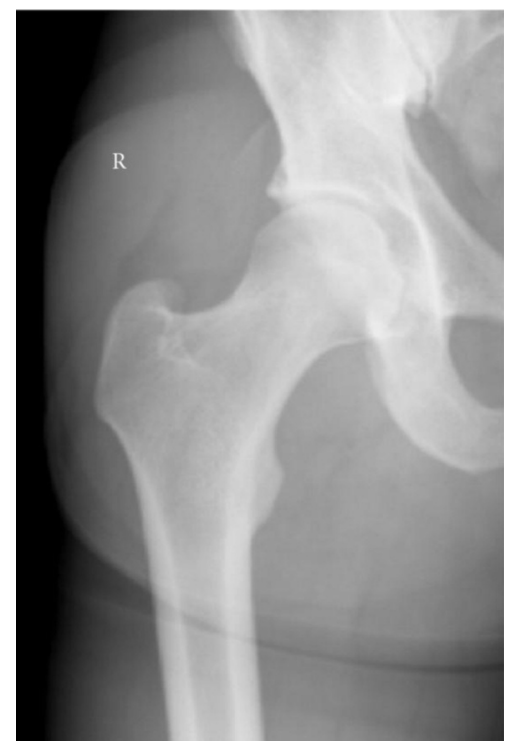

Fig. 2c: Radiograph of the hip at 1 year follow-up. injury, compounding, associated soft tissue (including vascular) injuries, $t$ age of the patient and delay in reduction $2,4,5$.

In our case, the wound seemed to be due to a progressive medial soft tissue injury secondary to the extreme external rotation and dislocation of the hip. This progression started with medial capsule disruption and joint dislocation and continued till the muscle and perineal skin gave way. The neurovascular structures were unharmed as the zone of injury passed between the femoral vessels and the sciatic nerve. Immediate reduction and adequate debridement with antibiotic coverage enabled the patient to have an uneventful recovery.
Eventual outcome following this rare injury depends on many factors such as initial cartilage damage, injury to the femoral head vasculature, and sometimes infection that are variables beyond the surgeon's control. Factors like timing and accuracy of the reduction are variables that can be positively affected by recognizing and treating the injury as an acute emergency. Thus, the aim of treatment should be avoidance of complications and then surprisingly good results can be achieved. 


\section{REFERENCES}

1. Khan SA, Sadiq SA, Abbas M, et al. Open anterior dislocation of the hip in a child. J Trauma. 2001; 51: 773-6.

2. Rafai M, Ouarab M, Largab A, et al. Open post-traumatic anterior luxation of the hip in children. Rev Chir Orthop Reparatrice Appar Mot 1995; 81(2): 178-81

3. Renato L. Open anterior dislocation of the hip in a child. Acta Orthop Scand. 1987; 58: 669-70.

4. Grundy M, Kumar N. Open anterior dislocation of the hip. Injury. 1982; 13: 315-6.

5. Lamberti PM, Rabin SI. Open anterior-inferior hip dislocation. J Orthop Trauma 2003. 17(1): 65-6. 